\title{
SARS-CoV-2 infection associated with spontaneous pneumothorax
}

\author{
Sagar Rohailla MD MSc, Najma Ahmed MD, Kevin Gough MD
}

Cite as: CMAJ 2020 May 11;192:E510. doi: 10.1503/cmaj.200609; early-released April 21, 2020

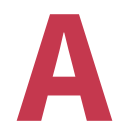

26-year-old man presented to the emergency department with a 3-day history of sudden-onset right-sided pleuritic chest pain and progressive shortness of breath. The patient had not travelled in the preceding 2 weeks, had no history of chest trauma, and had no history of fever, cough or other systemic symptoms. He was previously healthy and was not taking any medications. His cohabitant had a nonproductive cough for 1 week. The patient preferred not to disclose any further details.

On examination, our patient's vital signs included a normal temperature and blood pressure $(118 / 80 \mathrm{~mm} \mathrm{Hg})$ and heart rate of 122 beats/min. He had mild respiratory distress with normal oxygen saturation on ambient air. His body mass index was $17.6 \mathrm{~kg} / \mathrm{m}^{2}$. Results from blood work included lymphopenia (0.69 [normal $\left.1.0-3.2] \times 10^{9} / \mathrm{L}\right)$. Chest radiography showed a large right pneumothorax with complete collapse of the right lung without mediastinal shift (Figure 1A).

Because of the patient's respiratory symptoms, we did a nasopharyngeal swab for real-time reverse transcription polymerase chain reaction testing, which showed infection with severe acute respiratory syndrome coronavirus 2 (SARS-CoV-2). ${ }^{1}$ We placed a small catheter chest drain, which was later removed, with the clinician using full personal protective equipment precautions, including an N95 respirator. After 48 hours, our patient's pneumothorax and symptoms resolved (Figure 1B). He was discharged from hospital in stable condition, and he reported complete resolution of symptoms over video teleconferencing on postdischarge day 1 .

Our patient's symptoms did not follow the expected trajectory for coronavirus disease 2019 (COVID-19); however, he did have lymphopenia, which has been well-described in those with COVID-19. ${ }^{2}$ Spontaneous pneumothorax is commonly seen in taller young men of thin body habitus and is known to be a potential complication of infectious lung disease. ${ }^{3}$ An association between COVID-19 and spontaneous pneumothorax has been described elsewhere, but its prognostic significance is not known. ${ }^{4}$

\section{References}

1. Corman VM, Landt O, Kaiser M, et al. Detection of 2019 novel coronavirus (2019-nCoV) by real-time RT-PCR. Euro Surveill 2020;25. doi: 10.2807/1560-7917.ES.2020.25.3.2000045.

2. Huang C, Wang Y, Li X, et al. Clinical features of patients infected with 2019 novel coronavirus in Wuhan, China. Lancet 2020;395:497-506.

3. Sahn SA, Heffner JE. Spontaneous pneumothorax. N Engl J Med 2000;342:868-74.

4. Salehi S, Abedi A, Balakrishnan S, et al. Coronavirus disease 2019 (COVID-19): a systematic review of imaging findings in 919 patients. AJR Am J Roentgenol 2020 Mar. 14 [Epub ahead of print];1-7. doi: 10.2214/AJR.20.23034.

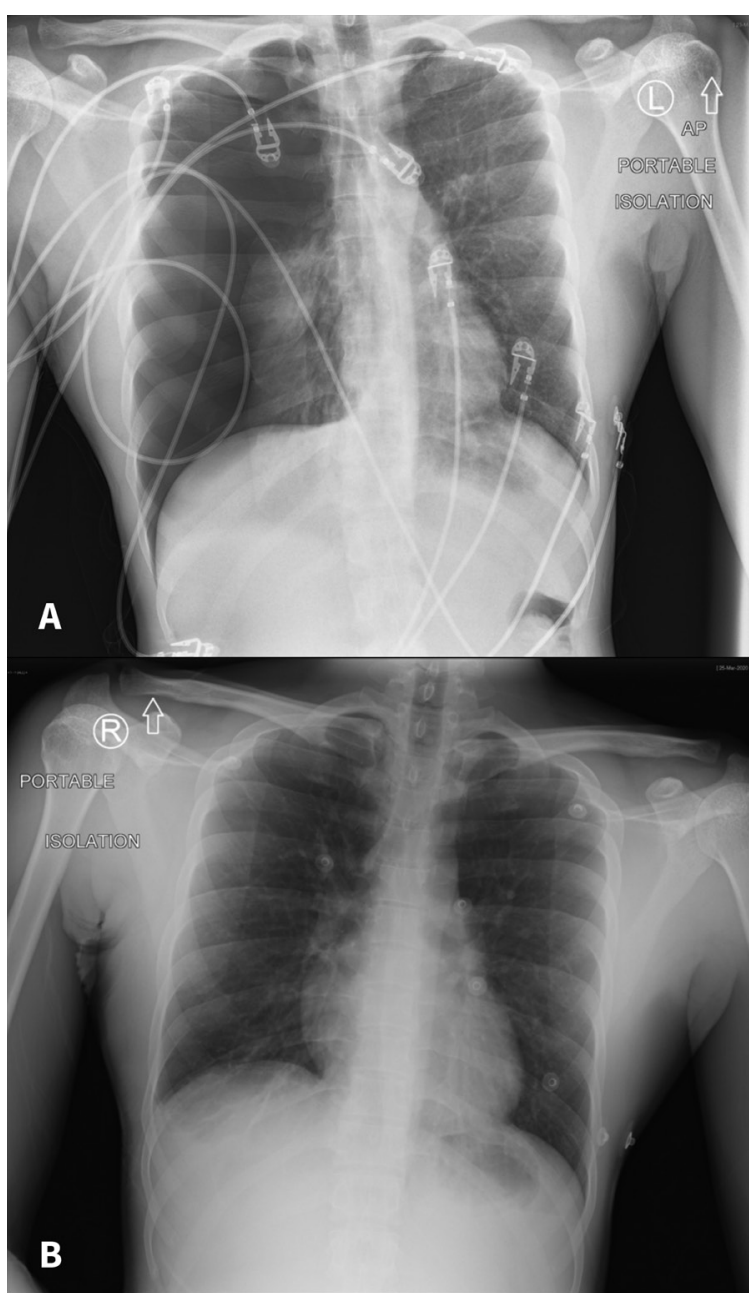

Figure 1: Chest radiograph of a 26-year-old man with severe acute respiratory syndrome coronavirus 2 infection showing (A) a large pneumothorax and complete collapse of the affected right lung obtained on admission to hospital. The left lung appears normal. (B) Expanded lung after removal of the pleural catheter. A trace right pleural effusion is visible with minor atelectasis. No pulmonary edema or consolidation is present.
Competing interests: None declared.

This article has been peer reviewed.
The authors have obtained patient consent.

Affiliation: St. Michael's Hospital, University of Toronto, Toronto, Ont.
Correspondence to: Kevin Gough,

Kevin.Gough@unityhealth.to 